\title{
Low back pain deprives the Japanese adult population of their quality of life: a questionnaire survey at five healthcare facilities in Japan
}

\author{
Machi Suka $\cdot$ Katsumi Yoshida
}

Received: 30 May 2007/ Accepted: 16 October 2007/Published online: 28 February 2008

(C) The Japanese Society for Hygiene 2008

\begin{abstract}
Objectives To estimate the degree to which low back pain (LBP) deprives the Japanese adult population of their quality of life (QOL) in terms of quality-adjusted life-years (QALYs).

Methods A questionnaire survey was conducted among participants of health examinations at five healthcare facilities in Japan. Age- and sex-specific mean values of the EQ-5D score were calculated for (1) those who reported LBP and interference with daily activities (IDA) due to the pain $(n=251)$, (2) those who reported LBP but no IDA $(n=955)$, and (3) those who reported no musculoskeletal pain $(n=2887)$. To estimate the loss of QALYs due to LBP in the Japanese adult population, we multiplied the age- and sex-specific mean differences of the EQ-5D scores between the LBP with (or without) IDA group and the no pain group by the corresponding age- and sex-specific numbers of people with LBP with (or without) IDA in Japan.

Results Among the entire Japanese adult population of 103 million people, $11,800,000 \quad(4,910,000$ men and $6,890,000$ women) were estimated to suffer from LBP, and 2,403,000 (976,000 men and 1,427,000 women) people were estimated to encounter IDA due to the pain. The loss of QALYs due to LBP in the Japanese adult population was estimated at 947,000 (9.18 per 1000 population). The loss of QALYs due to IDA in the LBP people was estimated at 139,000 (1.35 per 1000 population).
\end{abstract}

M. Suka $(\varangle) \cdot$ K. Yoshida

Department of Preventive Medicine,

St. Marianna University School of Medicine,

2-16-1 Sugao, Miyamae-ku, Kawasaki,

Kanagawa 216-8511, Japan

e-mail: suka@marianna-u.ac.jp
Conclusions The estimated loss of QALYs due to LBP suggests that LBP substantially deprives the Japanese adult population of their QOL.

Keywords Japan - Low back pain .

Quality adjusted life year - Quality of life

\section{Introduction}

Low back pain (LBP) is a major health and socioeconomic problem in Western countries [1]. Many people will experience one or more episodes of LBP in their life and, as such, LBP is associated with high healthcare costs, work absenteeism, and disablement.

Our previous study showed that $41.2 \%$ of the Japanese adult population suffers from musculoskeletal pain, with the lower back being the most common site of pain for both sexes [2]. As has been found in Western populations, LBP is likely to deprive the Japanese population of their quality of life (QOL). However, to date, few studies have evaluated the impact of LBP on QOL in a Japanese population. Although the Japanese lifestyle is becoming increasingly westernized, considerable gaps remain in terms of culture, customs, and body build between Japan and Western countries. It is therefore uncertain whether the results pertaining to Western populations are applicable to the Japanese population.

We conducted a questionnaire survey among participants in health examinations at five healthcare facilities in Japan with the aim of evaluating the impact of musculoskeletal pain on QOL. Based on the results of this questionnaire survey and the national statistical data, we then estimated to the degree to which LBP deprives the Japanese adult population of their QOL in terms of qualityadjusted life-years (QALYs). 


\section{Methods}

Questionnaire survey on musculoskeletal pain

A questionnaire survey was conducted in September and October 2005 at five healthcare facilities in Japan: (1) Niigata healthcare association (Niigata, Niigata prefecture), (2) Tsukuba multiphasic health examination center (Tsukuba, Ibaraki prefecture), (3) Omiya Kyoritsu hospital (Saitama, Saitama prefecture), (4) Seirei health examination center (Hamamatsu, Shizuoka prefecture), and (5) Wellness Sasaoka clinic (Fukuoka, Fukuoka prefecture). This questionnaire survey was approved by the ethics committee of St. Marianna University School of Medicine.

Approximately 1000 participants at each healthcare facility who were undergoing health examinations during the 2 months of the study period were asked to complete a questionnaire anonymously. The questionnaire consisted of two parts. In the first part, the participants marked the regions affected by musculoskeletal pain for more than 1 week during the last month on a drawing with predefined body regions (Fig. 1). Four different symbols were used to classify the types of pain as (1) treated or untreated and (2) with or without interference with daily activities (IDA) due to the pain. In the second part, the participants filled out the Japanese EQ-5D instrument [3] to assess their generic QOL. A total of 5652 people who agreed to participate in the questionnaire survey turned in their completed questionnaire then and there.
EQ-5D score

The EQ-5D instrument defines health according to five dimensions: (1) mobility, (2) self-care, (3) usual activities, (4) pain/discomfort, and (5) anxiety/depression. Each dimension consists of three levels of severity: (1) no problems, (2) some/moderate problems, and (3) extreme problems. A unique EQ-5D health state is defined by combining one level from each of the five dimensions ([3]; EuroQol Group, EQ-5D: http://www.euroqol.org).

Among the 5368 eligible people aged 20 years or older who completely filled out the Japanese EQ-5D instrument, 1206 (22.5\%) reported LBP and 2887 (54\%) reported no musculoskeletal pain. The EQ-5D health states were converted into a single index value - the EQ-5D score - by applying the EQ-5D value sets elicited from general population samples [3]. Age- and sex-specific mean values of the EQ-5D score were calculated for the following three groups: (1) those who reported LBP and IDA $(n=251$, LBP with IDA group), (2) those who reported LBP but no IDA ( $n=955$, LBP without IDA group), and (3) those who reported no musculoskeletal pain $(n=2887$, no pain group).

\section{Estimation of the number of LBP people}

Age- and sex-specific prevalence rates of LBP were derived from the 2004 National Life Survey [4]. To estimate the number of people with LBP in Japan, we
Fig. 1 Pain drawing with predefined body regions used in the questionnaire

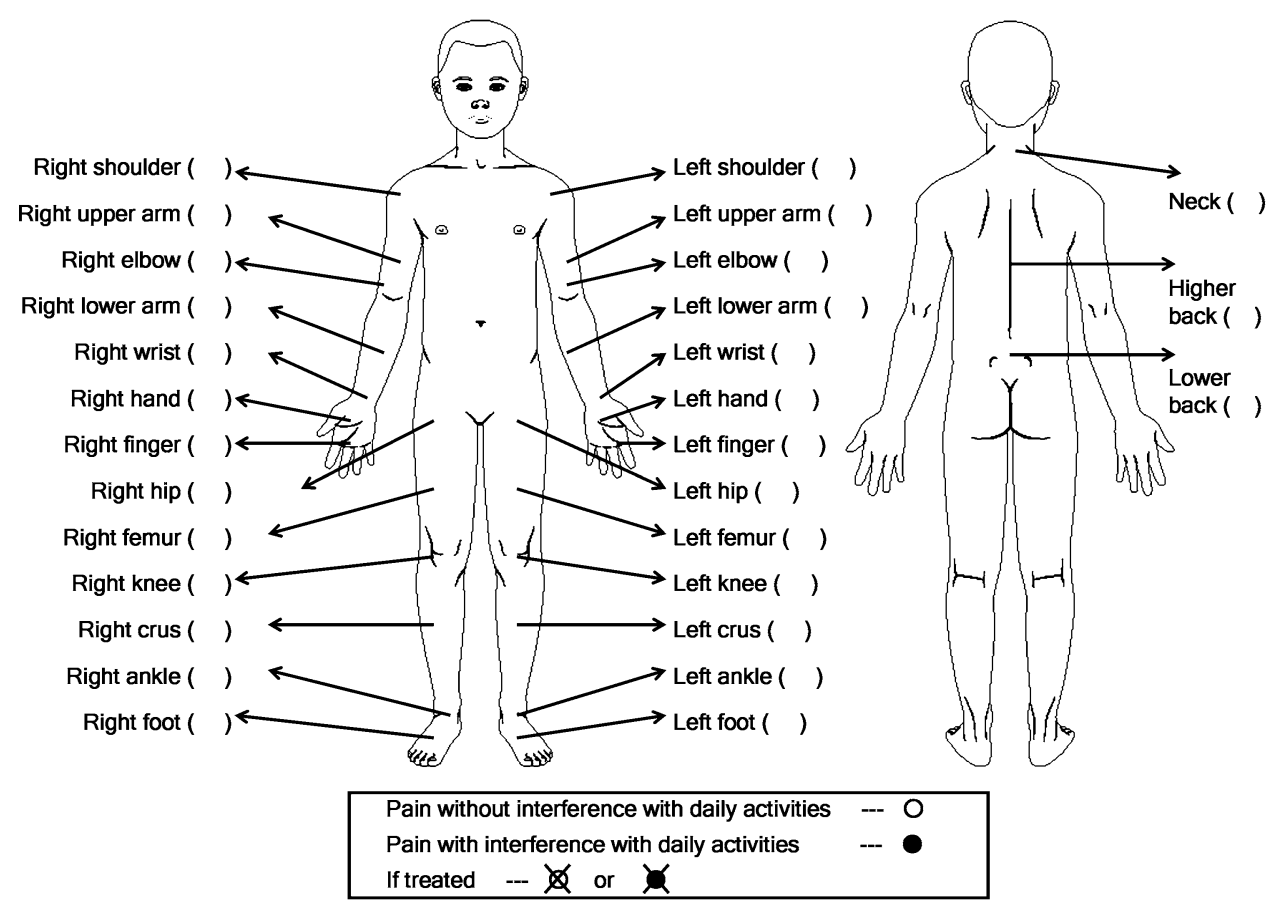


multiplied the age- and sex-specific prevalence rates of LBP by the corresponding age- and sex-specific population estimates as of October 1, 2004 [5]. Age- and sex-specific percentages of IDA in people with LBP that were derived from the questionnaire survey on musculoskeletal pain were used to estimate the number of people who encounter IDA due to the pain in Japan because these data were unavailable in the national statistical data.

\section{Estimation of the loss of QALYs due to LBP}

Quality-adjusted life-years are widely used as a measure of health outcomes as it quantifies the overall difference between two or more health states. The QALY combines the quantity and the quality of life in a single index value. A year of perfect health is considered to be equal to 1 QALY, while a year of ill health is discounted according to the degree to which the illness deprives the patients of their QOL $[6,7]$. To determine the QALY value, the years lived in a given health state are multiplied by the utility value of that health state. For example, a year lived in a health state with a utility value of 0.5 is equal to 0.5 QALYs, the same as half a year lived in perfect health. The utility values of the health states, ranging from 1 (indicating perfect health) to 0 (indicating death), can be estimated using a series of techniques, such as Standard Gamble, Time Trade-Off, and Rating Scale, or by means of health state scoring systems, such as the EQ-5D and Health Utilities Index. The EQ-5D instrument has produced a set of utilities values for 245 health states ([3]; EuroQol Group, EQ-5D: http://www. euroqol.org).

To estimate the loss of QALYs due to LBP in the Japanese adult population, we multiplied the age- and sexspecific mean differences of EQ-5D scores between the LBP with (or without) IDA group and the no pain group by the corresponding age- and sex-specific numbers of people with LBP and with (or without) IDA in Japan. To estimate the loss of QALYs due to IDA in people with LBP, the age- and sex-specific mean differences of EQ-5D scores between the LBP with and without IDA groups were multiplied by the corresponding age- and sex-specific numbers of people with both LBP and IDA in Japan.

\section{Statistical analyses}

Statistical analyses were performed with the Statistical Analysis Systems (SAS, ver. 8.2; SAS Institute, Cary, NC). The percentages of problems in five dimensions were compared using the chi-square test. The mean EQ-5D scores were compared by one-way analysis of variance. Tukey's HSD multiple comparisons were performed to calculate the difference between each pair of means with a $95 \%$ confidence interval $(\mathrm{CI})$.

\section{Results}

Table 1 shows the percentages of problems in five dimensions and EQ-5D scores in the questionnaire survey on musculoskeletal pain. The LBP with and without IDA groups reported more problems than the no pain group in all dimensions (mobility, self-care, usual activities, pain/ discomfort, and anxiety/depression). For the total subjects, a significant difference among the three groups was found in all dimensions except for self-care in women where the percentage of the problem was extremely low. The lowest EQ-5D score was observed in the LBP with IDA group, followed by the LBP without IDA group and the no pain group $(P<0.001)$. This trend was consistently found for all age groups and both sexes; the most striking difference was observed in the group of men aged $60+$ years.

Multiple comparisons showed that the LBP with and without IDA groups had a significantly lower EQ-5D score than the no pain group for all age groups and both sexes. The mean differences $(95 \% \mathrm{CI})$ in EQ-5D scores between the LBP with IDA group and the no pain group were 0.064 (0.027-0.100), 0.079 (0.063-0.094), and $0.220(0.178$ 0.263 ) for the age groups $20-39,40-59$, and $60+$ years, respectively, for men and $0.085(0.039-0.131), 0.120$ $(0.095-0.145)$, and $0.128(0.022-0.234)$ for the age groups $20-39,40-59$, and $60+$ years, respectively, for women. The mean differences $(95 \% \mathrm{CI})$ in EQ-5D scores between the LBP without IDA group and the no pain group were 0.034 (0.014-0.053), $0.052(0.043-0.061)$, and 0.038 (0.0150.062 ) for the age groups $20-39,40-59$, and $60+$ years, respectively, for men and $0.070(0.048-0.092), 0.067$ $(0.052-0.083)$, and $0.104(0.042-0.166)$ for the age groups 20-39, 40-59, and 60+ years, respectively, for women. These mean difference values were used to estimate the loss of QALYs due to LBP. Multiple comparisons also showed that the LBP with IDA group had a significantly lower EQ-5D score than the LBP without IDA group in the age groups $40-59$ and $60+$ years for men and $40-59$ years for women. The mean differences $(95 \% \mathrm{CI})$ in EQ-5D scores between the LBP with and without IDA groups were 0.030 ( -0.010 to 0.069$), 0.027(0.001-0.044)$, and $0.182(0.137-0.228)$ in the age groups $20-39,40-59$, and $60+$ years, respectively, for men and $0.015(-0.034$ to $0.064), 0.053(0.025-0.080)$, and $0.024(-0.089$ to 0.136$)$ in the age groups $20-39,40-59$, and $60+$ years, respectively, for women. These mean difference values were used to estimate the loss of QALYs due to IDA in people with LBP. 
Table 1 Percentages of problems in five dimensions and EQ-5D scores in the questionnaire survey on musculoskeletal pain

\begin{tabular}{|c|c|c|c|c|c|c|}
\hline \multirow[t]{3}{*}{ Age (years) } & \multicolumn{3}{|l|}{ Men } & \multicolumn{3}{|l|}{ Women } \\
\hline & \multirow[t]{2}{*}{ No pain } & \multicolumn{2}{|l|}{ Low back pain } & \multirow[t]{2}{*}{ No pain } & \multicolumn{2}{|l|}{ Low back pain } \\
\hline & & Without IDA & With IDA & & Without IDA & With IDA \\
\hline \multicolumn{7}{|l|}{ All } \\
\hline Number of subjects & 1835 & 585 & 151 & 1052 & 470 & 100 \\
\hline \multicolumn{7}{|l|}{ Percentages of problems ${ }^{\mathrm{a}}$} \\
\hline Mobility & 0.7 & 3.4 & $8.6 * * *$ & 1.0 & 5.4 & $8.0 * * *$ \\
\hline Self-care & 0.1 & 0.0 & $2.0 * * *$ & 0.2 & 0.5 & 1.0 \\
\hline Usual activities & 0.5 & 2.1 & $4.6^{* * *}$ & 0.5 & 4.1 & $13.0 * * *$ \\
\hline Pain/discomfort & 3.6 & 35.0 & $48.3 * * *$ & 7.2 & 49.7 & $67.0 * * *$ \\
\hline Anxiety/depression & 6.5 & 9.7 & $9.9^{*}$ & 10.4 & 18.4 & $20.0 * * *$ \\
\hline EQ-5D score (mean $\pm \mathrm{SD})$ & $0.91 \pm 0.04$ & $0.86 \pm 0.09$ & $0.82 \pm 0.16^{* * *}$ & $0.90 \pm 0.06$ & $0.83 \pm 0.14$ & $0.78 \pm 0.15^{* * *}$ \\
\hline \multicolumn{7}{|l|}{$20-39$} \\
\hline Number of subjects & 391 & 102 & 24 & 307 & 104 & 16 \\
\hline \multicolumn{7}{|l|}{ Percentages of problems ${ }^{\mathrm{a}}$} \\
\hline Mobility & 0.5 & 1.0 & 4.2 & 0.3 & 0.0 & 0.0 \\
\hline Self-care & 0.3 & 0.0 & 0.0 & 0.3 & 0.0 & 0.0 \\
\hline Usual activities & 1.3 & 2.0 & 4.2 & 0.3 & 2.3 & $6.3^{*}$ \\
\hline Pain/discomfort & 4.6 & 28.4 & $37.5 * * *$ & 6.2 & 44.3 & $43.8 * * *$ \\
\hline Anxiety/depression & 7.9 & 11.8 & 8.3 & 13.4 & 28.4 & $18.8^{* *}$ \\
\hline EQ-5D score (mean $\pm \mathrm{SD})$ & $0.90 \pm 0.06$ & $0.87 \pm 0.09$ & $0.84 \pm 0.14 * * *$ & $0.90 \pm 0.04$ & $0.83 \pm 0.12$ & $0.82 \pm 0.18^{* * *}$ \\
\hline \multicolumn{7}{|l|}{$40-59$} \\
\hline Number of subjects & 1253 & 408 & 109 & 643 & 309 & 72 \\
\hline \multicolumn{7}{|l|}{ Percentages of problems ${ }^{\mathrm{a}}$} \\
\hline Mobility & 0.5 & 3.7 & $6.4 * * *$ & 0.9 & 5.5 & $8.3 * * *$ \\
\hline Self-care & 0.0 & 0.0 & $0.9 * * *$ & 0.2 & 0.0 & 1.4 \\
\hline Usual activities & 0.4 & 1.7 & $2.8 * *$ & 0.6 & 3.8 & $9.7 * * *$ \\
\hline Pain/discomfort & 3.4 & 37.7 & $45.9 * * *$ & 7.3 & 51.5 & $68.1 * * *$ \\
\hline Anxiety/depression & 6.3 & 9.6 & $10.1 *$ & 9.6 & 15.6 & $20.8 * *$ \\
\hline EQ-5D score (mean \pm SD) & $0.91 \pm 0.04$ & $0.86 \pm 0.10$ & $0.83 \pm 0.14 * * *$ & $0.90 \pm 0.06$ & $0.83 \pm 0.11$ & $0.78 \pm 0.16^{* * *}$ \\
\hline \multicolumn{7}{|l|}{$60+$} \\
\hline Number of subjects & 191 & 75 & 18 & 102 & 57 & 12 \\
\hline \multicolumn{7}{|l|}{ Percentages of problems ${ }^{\mathrm{a}}$} \\
\hline Mobility & 2.1 & 5.3 & $27.8 * * *$ & 3.9 & 15.6 & $16.7 *$ \\
\hline Self-care & 0.0 & 0.0 & $11.0 * * *$ & 0.0 & 4.4 & 0.0 \\
\hline Usual activities & 0.0 & 4.0 & $16.7 * * *$ & 0.0 & 8.9 & $41.7 * * *$ \\
\hline Pain/discomfort & 3.1 & 29.3 & $77.8 * * *$ & 9.8 & 51.1 & $91.7 * * *$ \\
\hline Anxiety/depression & 4.7 & 8.0 & 11.1 & 5.9 & 13.3 & 16.7 \\
\hline EQ-5D score (mean $\pm \mathrm{SD})$ & $0.91 \pm 0.03$ & $0.87 \pm 0.07$ & $0.69 \pm 0.24 * * *$ & $0.90 \pm 0.07$ & $0.79 \pm 0.25$ & $0.77 \pm 0.07 * * *$ \\
\hline
\end{tabular}

IDA, interference with daily activities

$* P<0.05$; ** $P<0.01$; *** $P<0.001$ (difference among the three groups)

${ }^{a}$ People who reported some/moderate or extreme problems of the dimension were counted as having problems in the dimension

Table 2 shows the estimated number of LBP people and the estimated loss of QALYs due to LBP in the Japanese adult population. Among the entire Japanese adult population of 103 million people, 11,800,000 (4,910,000 men and 6,890,000 women) were estimated to suffer from LBP, and 2,403,000 (976,000 men and 1,427,000 women) were estimated to encounter IDA due to the pain. The prevalence rate of LBP was estimated at 114.4 (men 98.7, women 128.9) per 1000 population, with a significant increase with age; the prevalence rate in the $60+$ year age group was 2.7fold that in the age group 20-39 years for both sexes. The loss of QALYs due to LBP in the Japanese adult population 
Table 2 Burden of low back pain in the Japanese adult population

\begin{tabular}{|c|c|c|c|c|c|c|}
\hline \multirow[t]{3}{*}{ Age (years) } & \multicolumn{3}{|l|}{ Men } & \multicolumn{3}{|l|}{ Women } \\
\hline & \multirow[t]{2}{*}{ Prevalence $\times 1000$} & \multicolumn{2}{|l|}{ Loss of QALYs } & \multirow[t]{2}{*}{ Prevalence $\times 1000$} & \multicolumn{2}{|l|}{ Loss of QALYs } \\
\hline & & Due to pain & Due to IDA & & Due to pain & Due to IDA \\
\hline \multicolumn{7}{|l|}{ All } \\
\hline Total & 4909.8 (98.7) & $299862(6.03)$ & - & 6890.5 (128.9) & $647423(12.11)$ & - \\
\hline Pain with IDA & 976.8 (19.6) & - & $93627(1.88)$ & $1426.6(26.7)$ & - & $45437(0.85)$ \\
\hline \multicolumn{7}{|l|}{ 20-39 } \\
\hline Total & $1018.3(57.4)$ & 40247 (2.27) & - & $1242.6(72.1)$ & $89622(5.20)$ & - \\
\hline Pain with IDA & 193.5 (10.9) & - & $5727(0.32)$ & $191.4(11.1)$ & - & $2870(0.17)$ \\
\hline \multicolumn{7}{|l|}{$40-59$} \\
\hline Total & $1670.8(96.5)$ & $96331(5.57)$ & - & 1980.6 (113.9) & $157321(9.04)$ & - \\
\hline Pain with IDA & $352.5(20.4)$ & - & $9448(0.55)$ & $461.5(26.5)$ & - & 24227 (1.39) \\
\hline \multicolumn{7}{|l|}{$60+$} \\
\hline Total & $2220.7(151.1)$ & $163284(11.11)$ & - & 3667.4 (194.8) & $400480(21.27)$ & - \\
\hline Pain with IDA & $430.8(29.3)$ & - & $78452(5.34)$ & 773.8 (41.1) & _- & $18340(0.97)$ \\
\hline
\end{tabular}

QALYs, Quality-adjusted life-years; IDA, interference with daily activities

Values in parentheses are prevalence and loss of QALYs per 1000 population

was estimated to be 947,000 (9.18 per 1,000 population). The loss of QALYs due to IDA in the LBP people was estimated at 139,000 (1.35 per 1000 population).

\section{Discussion}

This study is the first attempt to estimate the loss of QALYs due to LBP in the Japanese adult population. QALYs are widely used as a measure of health outcomes as it quantifies the overall difference between two or more health states [6, 7]. The measurement of QALYs provides useful information for underpinning resource allocation decisions [8]. Despite its higher prevalence, LBP has not received the same level of attention by health professionals in Japan as cancers and cardiovascular diseases. However, the estimated loss of QALYs due to LBP suggests that LBP substantially deprives the Japanese adult population of their QOL. Ohkusa reported that the Japanese people may be willing to pay one million YEN to improve 1 QALY [9]. When this value applies to the estimated loss of QALYs due to LBP, the improvement of QOL in people with LBP may be worth an investment of 947 thousand million YEN (equivalent to $0.2 \%$ of the gross domestic product; Cabinet Office, Government of Japan. Quarterly estimates of GDP: http://www.esri.cao.go.jp/en/sna/menu.html) in Japan.

To our knowledge, this is the first study that has evaluated the impact of musculoskeletal pain on QOL in the Japanese adult population. We previously conducted a questionnaire survey to estimate the prevalence of musculoskeletal pain in the Japanese adult population [2], but the measurement of QOL was not included in the survey questions. The result of the questionnaire survey on musculoskeletal pain (Table 1) showed that people with LBP reported more health problems than those without musculoskeletal pain in all dimensions (mobility, self-care, usual activities, pain/discomfort, and anxiety/depression). Note that significant differences were found in both physical and psychological dimensions. Similar results have been found for population samples from other countries [10-13]. One reasonable explanation for the relatively poorer health of people with LBP is that LBP induces stress, distress, anxiety, and/or depression along with dysfunction. On the other hand, a review of literature suggested that psychological problems may be associated with the onset and development of LBP [14]. Because of the cross-sectional design of our study, our results do not allow us to determine the causal relationship between LBP and psychological problems. However, it is important to note that LBP people may be damaged both physically and psychologically.

The use of the EQ-5D instrument makes it possible to express each health state in a single index value, that is, EQ-5D score. The EQ-5D instrument is widely used to compare generic QOL of different health state groups. Many investigators have reported that EQ-5D scores decrease with increasing prevalence of chronic conditions in general population samples. In a Swedish population, people with LBP showed lower EQ-5D scores than those with hypertension, diabetes, asthma, and ischemic heart disease; next to depression, LBP was associated with the greatest loss of QOL $[10,11]$. Similar results were obtained 
in Finnish and U.S. populations [15, 16]. Unfortunately, data on EQ-5D scores for people with chronic conditions are scarce in Japan. However, it is unlikely that the relative importance of each chronic condition in the Japanese population is completely different from that in Western populations. Further studies may be required to compare the impacts of chronic conditions on QOL in the Japanese adult population, which may contribute to optimal resource allocation decisions.

This study was not without its limitations. First, both acute and chronic cases were mixed together in the LBP people of this study. Due to the cross-sectional design, it was difficult to determine the course and prognosis of LBP. The loss of QALYs due to LBP may be overestimated when some of the LBP people eventually completely recover from the pain and disabilities. However, the DMC3 study, which is a population-based study carried out in the Netherlands, showed that $95 \%$ of LBP people described the pain as continuous or recurrent [17]. Pengel et al. [18] found that most people with acute LBP experienced rapid improvements in pain and disabilities within 1 month but that the cumulative risks of at least one recurrence within 3 and 12 months were 26\% (95\% CI: 19-34) and 73\% (95\% CI: 59-88), respectively. Hestbaek et al. [19] showed that the percentage of people with LBP who still experienced pain after 12 months was 42-75\% (average 62\%). Consequently, the estimated loss of QALYs due to LBP may be somewhat higher but not so different from the actual value. Second, participants in health examinations were recruited to the questionnaire survey on musculoskeletal pain. Generally speaking, participants in health examinations are rather healthy people, excluding bedridden patients. They are more likely to be aware of their own health and sensitive to impairment of QOL. The impact of LBP on QOL (Table 1) may have been affected by the selection bias. Third, comorbidity was not adjusted in the estimation of the loss of QALYs due to LBP. Many investigators have reported that comorbidity has a significant negative effect on the QOL of people with LBP [10-12]. The loss of QALYs due to LBP may have been overestimated when some of the people with LBP had chronic conditions, particularly depression and other musculoskeletal disorders. The QOL of LBP people may possibly be affected by socio-economic factors, such as low educational level, manual occupation, and low income [10-12]. Further studies may be required to explain the impact of LBP on QOL in detail.

In conclusion, the estimated loss of QALYs due to LBP suggests that LBP substantially deprives the Japanese adult population of their QOL. Although LBP has not received the same level of attention by health professionals in Japan as a number of other serious conditions, the control of LBP should not be bypassed in attempts to improve the QOL. Low back pain is typically classified as being "specific" or "non-specific". Approximately $90 \%$ of all people with LBP will have non-specific LBP, which is a diagnosis based on the exclusion of specific pathology [20]. Health professionals should pay attention to the implementation of effective preventive and therapeutic interventions for nonspecific LBP [20].

Acknowledgments This study was supported by the 2005-2006 Health and Labour Sciences Research Grant (Research on Prevention and Treatment for Immune Allergic Diseases) from the Japanese Ministry of Health Labour and Welfare. We would like to thank all of the staff of Niigata healthcare association, Tsukuba multiphasic health examination center, Omiya Kyoritsu Hospital, Seirei health examination center, and Wellness Sasaoka Clinic for their help and assistance of the questionnaire survey.

\section{References}

1. van Tulder M, Koes B, Bombardier C. Low back pain. Best Pract Res Clin Rheumatol. 2002;16:761-75.

2. Suka M, Yoshida K. Musculoskeletal pain in Japan: prevalence and interference with daily activities. Mod Rheumatol. 2005; 15:41-7.

3. Ikegami N, Fukuhara S, Shimozuma K, Ikeda S, editors. Handbook for assessment of QOL (in Japanese). Tokyo: Igaku-Shoin; 2001.

4. Japanese Ministry of Health, Labour, and Welfare, Minister's Secretariat, Department of Statistics and Information (eds.). The 2004 National Life Survey (in Japanese). Tokyo: Health and Welfare Statistics Association; 2006.

5. Japanese Ministry of Internal Affairs and Communications, Statistics Bureau. Current Population Estimates as of October 1, 2004. Available at: http://www.stat.go.jp/english/data/jinsui/2004np/ index.htm.

6. Petrou S, Renton A. The QALY: a guide for the public health physician. Public Health. 1993;107:327-36.

7. Sassi F. Calculating QALYs, comparing QALY and DALY calculations. Health Policy Plan. 2006;21:402-8.

8. Robinson R. Cost-utility analysis. Br Med J. 1993;307:859-62.

9. Ohkusa Y. Empirical research for the critical value of expenditure per QALY (in Japanese). Iryo Syakai. 2003;13:121-9.

10. Burstrom K, Johannesson M, Diderichsen F. Health-related quality of life by disease, socio-economic group in the general population in Sweden. Health Policy. 2001;55:51-69.

11. Burstrom K, Johannesson M, Diderichsen F. Swedish population health-related quality of life results using the EQ-5D. Qual Life Res. 2001;10:621-35.

12. Salaffi F, De Angelis R, Stancati A, Grassi W. Health-related quality of life in multiple musculoskeletal conditions: a crosssectional population based epidemiological study, II, the MAPPING study. Clin Exp Rheumatol. 2005;23:829-39.

13. Oksuz E. Prevalence, risk factors, and preference-based health states of low back pain in a Turkish population. Spine. 2006;31:E968-72.

14. Linton SJ. A review of psychological risk factors in back and neck pain. Spine. 2000;25:1148-56.

15. Saarni SI, Harkanen T, Sintonen H, Suvisaari J, Koskinen S, Aromaa A, et al. The impact of 29 chronic conditions on healthrelated quality of life: a general population survey in Finland using 15D and EQ-5D. Qual Life Res. 2006;15:1403-14. 
16. Ko Y, Coons SJ. Self-reported chronic conditions and EQ-5D index scores in the US adult population. Curr Med Res Opin. 2006;22:2065-71.

17. Picavet HSJ, Schouten JSAG. Musculoskeletal pain in Netherlands: prevalence, consequence, and risk groups, the DMC3 study. Pain. 2003;102:167-78.

18. Pengel LHM, Herbert RD, Maher CG, Refshauge KM. Acute low back pain: systematic review of its prognosis. $\mathrm{Br}$ Med $\mathrm{J}$. 2003;327:323-7.
19. Hestbaek L, Leboeuf-Yde C, Manniche C. Low back pain: what is the long-term course? a review of studies of general patient populations. Eur Spine J. 2003;12:149-65.

20. Koes BW, van Tulder MW, Thomas S. Diagnosis and treatment of low back pain. Br Med J. 2006;332:1430-4. 